intima-media thickness (CIMT), a technique that can identify early-stage atherosclerosis, to show an association between IBD and increased atherosclerosis risk. They also demonstrated independent correlations between CIMT, age, and increased levels of plasma homocysteine, a variant of the amino acid cysteine present in excess in patients with IBD.

A series of 52 patients with IBD, <45 years old and without cardiovascular disease or atherosclerosis risk factors, as well as 20 healthy controls with no history of inflammatory disease, underwent carotid ultrasonography and assessment of plasma homocysteine levels. A detailed clinical history was also collected, including use of medications.

Compared with controls, patients with IBD had significantly increased CIMT (mean $0.63 \mathrm{~mm}$ versus $0.53 \mathrm{~mm}, P=0.008$ ) and plasma homocysteine levels (mean $9.4 \mu \mathrm{M}$ versus $7.1 \mu \mathrm{M}, P=0.003$ ). No atherosclerotic lesions $>2 \mathrm{~mm}$ were identified in either group. Interestingly, the difference in CIMT between IBD patients not taking the anti-tumor-necrosisfactor monoclonal antibody infliximab and controls was significant (mean $0.65 \mathrm{~mm}$ versus $0.53 \mathrm{~mm}, P=0.003$ ), whereas there was no significant difference in CIMT between IBD patients taking the drug and controls, suggesting that infliximab might be protective against atherosclerosis in this setting.

Rebecca Doherty

Original article Papa A et al. (2005) Increased carotid intima-media thickness in patients with inflammatory bowel disease. Aliment Pharmacol Ther 22: 839-846

\section{Laparoscopic ileal-pouch-anal anastomosis is safe and effective}

Ileal-pouch-anal anastomosis (IPAA) is the most commonly performed surgery for ulcerative colitis and familial adenomatous polyposis. Although originally performed as open surgery, a laparoscopic approach has demonstrated short-term benefits such as shorter hospitalization, reduced narcotic use and diminished postoperative pain. Few studies have examined how the longer-term outcomes of laparoscopic IPAA compare with those of open IPAA. In this prospective, single-center study, Larson et al. found that a laparoscopic approach does not compromise intermediate-term function or quality of life when compared with open surgery.

A questionnaire was used to collect functional and quality-of-life data from 33 patients who had undergone laparoscopic IPAA surgery $>1$ year previously. Each of these patients was matched for age, sex, BMI, diagnosis, and year of operation ( \pm 2 years) with a patient who had undergone open IPAA surgery. Median time of follow-up for both groups was 13 months. Functional outcome and quality-oflife measures did not differ between the two groups 1 year after surgery.

Although the quality-of-life questionnaire used in this study has been used by the authors in their practice for $>20$ years, it should be noted that it has not been formally validated. Future studies should use a validated assessment tool, and look at longer-term outcomes, to confirm that the laparoscopic approach is equivalent to traditional, open IPAA surgery.

Katherine Sole

Original article Larson DW et al. (2005) Laparoscopic-assisted $v s$. open ileal pouch-anal anastomosis: functional outcome in a case-matched series. Dis Colon Rectum 48: 1845-1850

\section{Outcomes of living, adult, right-lobe liver donors}

Since its inception in 1998, the use of livingdonor liver transplantation (LDLT) has rapidly increased. This technique, however, raises ethical issues regarding the impact of the procedure on the donor's quality of life and necessitates ongoing study of donor characteristics and morbidity. Two centers have analyzed their experience of using living, adult, rightlobe liver donors.

Shah and colleagues reported on 101 consecutive donors and found an overall complication rate of $37 \%$, with most events occurring within 30 days of the procedure. Beyond 30 days, $12 \%$ of donors were readmitted for complications, but no long-term complications became apparent during follow-up and all have returned to full activities. Interestingly, since the start of the LDLT program at these authors' center, there has been a significant decrease in the associated morbidity rate.

Verbesey and co-workers enrolled 47 donors, who self-reported on the effects of the procedure on their quality of life before the procedure and at five time points over the 